\title{
Parity Realization in Lattice QCD with Ginsparg-Wilson Fermions
}

\author{
V. Azcoiti, E. Follana, A. Vaquero, \\ Universidad de Zaragoza \\ G. Di Carlo \\ Laboratorio Nazionale di Gran Sasso
}

November 13, 2018

\begin{abstract}
The Vafa-Witten arguments on the realization of parity and flavour symmetries in the QCD vacuum do not apply to two lattice regularizations of QCD which are able to reproduce the chiral anomaly: Wilson fermions and Ginsparg-Wilson fermions. We show here how using the last regularization one can get, from first principles, that the more standard order parameters for these symmetries take a vanishing vacuum expectation value for any number of flavours, if quarks have a non-vanishing mass.
\end{abstract}

\section{Introduction}

Since the very beginning of the formulation of QCD as the gauge theory which describes the strong interaction between elementary particles, the understanding of the realization of symmetries in the QCD vacuum has been an important issue. During the 80's, Vafa and Witten gave arguments against spontaneous breaking of parity [1] and vector-like global symmetries [2] in vector-like theories such as QCD, and in principle, this seemed to settle the matter; however, these arguments were not as useful as expected. Indeed some years after the publication of 1, several articles appeared [3, 4, 5, 6], calling into question the validity of the paper. The fact that the issue is still open twenty years after the publication of the first paper is indicative of the complexity of the subject. Regarding vector-like symmetries such as flavour or baryon number conservation, it must be remarked that the Vafa-Witten theorem [2] is not applicable neither to the Ginsparg-Wilson regularization nor to one of the most used fermionic regularizations on the lattice for QCD, i.e., Wilson fermions. In the Ginsparg-Wilson case the theorem does not apply because even if the integration measure is positive definite, the other essential ingredient in the proof in [2, the anticommutation of the Dirac operator with $\gamma_{5}$, is not realized. For the case of Wilson fermions neither of the two assumptions in 2, positivity of the integration measure and anticommutation of the Dirac operator with $\gamma_{5}$, are fulfilled, the first of the two failing for an odd number of flavours. Indeed there exists a region of the parameters space where parity and flavour symmetries are spontaneously broken: the well known Aoki phase [7, 8, and even a more complex phase structure for 
lattice QCD with Wilson fermions has been recently suggested 9, 10. In the end, a theoretical proof of the realization of symmetries of QCD is still lacking.

The standard wisdom is that Vafa and Witten theorems fail, when applied to Wilson fermions, due to the existence of exceptional configurations which have a non-vanishing weight in the Aoki phase. Outside this phase, and in particular in the physical region near the continuum limit, the exceptional configurations would be suppressed and then parity and flavour symmetries would be restored in the QCD vacuum. Following this wisdom, it would be very convenient to choose a 'small eigenvalue free' regularization of QCD. It happens that GinspargWilson fermions fulfill this requirement.

In this paper we will show how the probability distribution function (p.d.f.) of all the standard fermion bilinear order parameters 11 for parity and flavour symmetry, as well as the p.d.f. of the topological charge density, are trivial in lattice QCD with Ginsparg-Wilson fermions of non-vanishing mass. The essential ingredient in what concerns the proof of parity realization in the vacuum will be hermiticity. The rest of this article is organized as follows. In section 2 we recall briefly the main features of Ginsparg-Wilson fermions and analyze the one-flavour case. Section 3 generalizes the results of the previous section to an arbitrary number of flavours, and shows how, contrary to the Wilson fermions regularization, the two flavour model has not an Aoki phase in any region of the parameter space. Section 4 contains a short discussion on the expectations about the realization of these symmetries in the chiral limit. In this limit the proof developed in the previous sections does not apply, and therefore we will argue following the standard wisdom. Hence, the content of that section is rather speculative. Last section contains our conclusions.

\section{Ginsparg-Wilson fermions on the lattice}

Years ago Ginsparg and Wilson (G-W) [12] suggested, in order to avoid the Nielsen and Ninomiya non go theorem [13, 14 and to preserve chiral symmetry on the lattice, to require the following condition for the inverse Dirac operator

$$
\gamma_{5} D^{-1}+D^{-1} \gamma_{5}=2 a R \gamma_{5},
$$

where $\mathrm{a}$ is the lattice spacing and $\mathrm{R}$ is a local operator. Accordingly $\mathrm{D}$ should satisfy, instead of the standard anticommutation relation of the continuum formulation, the Ginsparg-Wilson relation

$$
\gamma_{5} D+D \gamma_{5}=2 a D R \gamma_{5} D .
$$

Fifteen years after this proposal, Hasenfratz [15, and Neuberger 16, 17, found that the fixed point action for QCD and the overlap fermions satisfy respectively the Ginsparg-Wilson relation, the last with $\mathrm{R}=1 / 2$. Furthermore,

Hasenfratz, Laliena and Niedermayer [18, realized that Ginsparg-Wilson fermions have nice chiral properties, allowing us to establish an exact index theorem on the lattice. Indeed if we define a local density of topological charge as

$$
q(x)=\operatorname{aRTr}\left(\gamma_{5} D(x, x)\right)
$$

one gets a topological charge

$$
Q=a R T r\left(\gamma_{5} D\right)
$$


which is a topological invariant integer that approaches the continuum topological charge in the continuum limit [19, 20, 21, 22, 23.

Finally by replacing the G-W Dirac operator $D+m$ by

$$
\Delta+m=\left(1-\frac{a m}{2}\right) D+m
$$

in order to define an unsubtracted proper order parameter 24

$$
\bar{\psi}\left(1-\frac{a D}{2}\right) \psi,
$$

it is easy to see 25] that the G-W fermionic action possesses an exact symmetry which is anomalous for the flavour singlet transformations, but exact for the flavour non-singlet case; a property which allows us to introduce also a $\theta$ parameter in the G-W action, as in the continuum.

In this paper we will work with $\mathrm{G}-\mathrm{W}$ fermions that realize relation (2) with $R=1 / 2$ (overlap fermions are a particular case of that), and with the massive Dirac operator (5) associated to the unsubtracted chiral order parameter (6).

We derive in appendix $\mathrm{A}$ some properties of the spectrum of $\Delta+m$ and related operators that will be useful in the following.

\section{The one flavour case}

Let us start by considering the one flavour case. We will make use of the formalism developed in [6, 11]. First, we analyze the standard bilinear $O=i \bar{\psi} \gamma_{5} \psi$. Our basic tool is the function $P(q)$, which is the generating function for the moments of $\left.O: P(0)=1, P^{\prime}(0)=i<O>, \cdots, P^{n}(0)=(i)^{n}<O^{n}\right\rangle$, where the average is the lattice gauge theory average with the full action. We can compute $P(q)$ as 6 ]

$$
P(q)=\left\langle\frac{\operatorname{det}\left(\Delta+m+\frac{q}{V} \gamma_{5}\right)}{\operatorname{det}(\Delta+m)}\right\rangle=\left\langle\frac{\operatorname{det}\left(H+\frac{q}{V}\right)}{\operatorname{det}(H)}\right\rangle
$$

where $\mathrm{V}$ denotes the size of the matrix $\Delta$ (that is, up to a constant, the number of points in the lattice), $H=\gamma_{5}(\Delta+m)$, and the averages are now taken with the effective gauge theory measure obtained after integrating out the fermion fields

$$
[d A] e^{-S_{Y M} \operatorname{det}(\Delta+m)}
$$

We denote by $\mu_{j}$ the eigenvalues of $H$. Then we have for $P(q)$

$$
P(q)=\left\langle\frac{\prod_{j}\left(\mu_{j}+\frac{q}{V}\right)}{\prod_{j}\left(\mu_{j}\right)}\right\rangle=\left\langle\prod_{j}\left(1+\frac{q}{\mu_{j} V}\right)\right\rangle
$$

In appendix $\AA$ we show that $m \leq\left|\mu_{j}\right|$, so everything is well defined here as long as $m>0$. Expanding the product we rewrite $P(q)$ as

$$
P(q)=\sum_{k=0}^{V} q^{k}\left\langle\frac{1}{V^{k}} \sum_{\left(j_{1}, \cdots, j_{k}\right)} \frac{1}{\mu_{j_{1}}, \cdots, \mu_{j_{k}}}\right\rangle
$$


The sum in (10) is taken over all different combinations of $\mathrm{k}$ indices, so we can rearrange it as

$$
\frac{1}{V^{k}} \sum_{\left(j_{1}, \cdots, j_{k}\right)} \frac{1}{\mu_{j_{1}}, \cdots, \mu_{j_{k}}}=\frac{1}{k !}\left(\frac{1}{V} \sum_{j} \frac{1}{\mu_{j}}\right)^{k}+\mathcal{O}\left(\frac{1}{V}\right)
$$

But we show in appendix $\AA$ that the $\mu$ come in pairs $\pm \mu$, except maybe for the ones corresponding to chiral modes. Therefore most of the terms in (11) cancel, and we are left with the contribution coming from the chiral modes:

$$
\frac{1}{V} \sum_{j} \frac{1}{\mu_{j}}=\frac{1}{V}\left(\frac{1}{m}\left(n^{+}-n^{-}\right)+\frac{a}{2}\left(n^{\prime+}-n^{-}\right)\right)=\left(\frac{a m}{2}-1\right) \frac{Q}{m V}
$$

Putting everything together, we have, for non-zero mass

$$
P(q)=\sum_{k=0}^{V} \frac{q^{k}}{k !}\left(\frac{a m}{2}-1\right)^{k} \frac{1}{m^{k}}\left\langle\left(\frac{Q}{V}\right)^{k}\right\rangle+\mathcal{O}\left(\frac{1}{V}\right)
$$

We see that in the thermodynamic limit the only dependence of $P(q)$ on the gauge field is through the topological charge. From (13) the moments of the probability distribution of $O=i \bar{\psi} \gamma_{5} \psi$ are related to the ones of the density of topological charge $\frac{Q}{V}$ through

$$
\left\langle\left(i \bar{\psi} \gamma_{5} \psi\right)^{n}\right\rangle=(-i)^{n}\left(\frac{a m}{2}-1\right)^{n} \frac{1}{m^{n}} \frac{\left\langle Q^{n}\right\rangle}{V^{n}}+\mathcal{O}\left(\frac{1}{V}\right)
$$

All the odd moments vanish by symmetry. The first non-trivial moment is the second one

$$
\left\langle\left(i \bar{\psi} \gamma_{5} \psi\right)^{2}\right\rangle=-\left(\frac{a m}{2}-1\right)^{2} \frac{1}{m^{2}}\left\langle\left(\frac{Q}{V}\right)^{2}\right\rangle+\mathcal{O}\left(\frac{1}{V}\right)
$$

At this point we require that $i \bar{\psi} \gamma_{5} \psi$ be an hermitian operator. This is a simple requirement, but with important consequences: The expectation value of the square of an hermitian operator must be positive, but from (15), in the thermodynamic limit it is manifestly negative. The only way to fulfill both requirements at the same time is for the second moment to vanish,

$$
\lim _{V \rightarrow \infty}\left\langle\left(\frac{Q}{V}\right)^{2}\right\rangle=0
$$

But then the probability distribution of the density of topological charge, $Q / V$, must go to a delta at the origin

$$
\lim _{V \rightarrow \infty} p\left(\frac{Q}{V}\right)=\delta\left(\frac{Q}{V}\right)
$$

and all the higher moments of both $i \bar{\psi} \gamma_{5} \psi$ and $\frac{Q}{V}$ vanish as well, therefore parity is not broken in lattice QCD with one flavour of Ginsparg-Wilson fermions, at least for the standard order parameter $i \bar{\psi} \gamma_{5} \psi$. 
Let us consider now the case of the unsubstracted order parameter,

$$
i \bar{\psi} \gamma_{5}\left(1-\frac{a D}{2}\right) \psi
$$

We have

$$
P(q)=\left\langle\frac{\operatorname{det}\left(\Delta+m+\frac{q}{V} \gamma_{5}\left(1-\frac{a D}{2}\right)\right)}{\operatorname{det}(\Delta+m)}\right\rangle
$$

Let's prove that only the zero modes of $D$ contribute to (19). The matrix corresponding to the numerator is block-diagonal, and the contribution to the ratio coming from a pair of complex eigenvalues of $D$ is of the form (from (63) in appendix $\mathrm{A}$ )

$$
1-\alpha \frac{q^{2}}{V^{2}}
$$

with $|\alpha| \leq m^{-2}$ (64). Therefore the contribution to $P(q)$ corresponding to complex eigenvalues comes from the factor $\prod_{j}\left(1-\alpha_{j} \frac{q^{2}}{V^{2}}\right)$, where the product extends over all pairs of complex eigenvalues. Expanding this product, we have for the coefficient corresponding to $q^{2 k}$

$$
\left|\frac{1}{V^{2 k}} \sum_{\left(j_{1}, \ldots, j_{k}\right)} \alpha_{j_{1}} \cdots \alpha_{j_{k}}\right| \leq \frac{1}{V^{2 k}}\left(\sum_{j}\left|\alpha_{j}\right|\right)^{k} \leq \frac{V^{k} m^{-2 k}}{V^{2 k}}=\frac{m^{-2 k}}{V^{k}}
$$

Thus the contribution from the complex eigenvalues is of order $1+\mathcal{O}\left(\frac{1}{V}\right)$, that is, just 1 in the thermodynamic limit.

The chiral modes of $D$ with $\lambda=\frac{2}{a}$ contribute also 1 (65). The zero modes of $D$, on the other hand, give a non-trivial contribution:

$$
P(q)=\left(1+\frac{q}{m V}\right)^{n^{+}}\left(1-\frac{q}{m V}\right)^{n^{-}}
$$

If $n^{+}<n^{-}(Q>0)$,

$$
P(q)=\left(1-\frac{q^{2}}{m^{2} V^{2}}\right)^{n^{+}}\left(1-\frac{q}{m V}\right)^{Q}
$$

A similar expression is valid for $n^{+}>n^{-}$. We can argue essentially as in (21) to see that the first factor in (23) goes to 1 in the thermodynamic limit. Therefore we obtain the final result (valid for arbitrary values of $n^{+}, n^{-}$) when $V \rightarrow \infty$

$$
P(q)=\left(1-\operatorname{sign}(Q) \frac{q}{m V}\right)^{|Q|}
$$

All odd moments vanish as before because of (finite-volume) parity symmetry. The even moments also vanish because they are trivially related to the ones in (13), as can be seen easily by expanding (24). In consequence we see that parity is not broken for the unsubstracted order parameter either.

It is interesting to give a more physical argument that uses only the vanishing of the second moment. In fact if parity were spontaneously broken we would expect two degenerate vacua $\alpha$ and $\beta$, since parity is a $Z_{2}$ symmetry. Let $z_{\alpha}$ be 
a complex number which give us the mean value of the pseudoscalar $P$ in the $\alpha$ state

$$
\langle P\rangle_{\alpha}=z_{\alpha}
$$

then we have

$$
\langle P\rangle_{\beta}=-z_{\alpha} .
$$

Since $P^{2}$ is parity invariant, it will take the same mean value in the two states and making use of the cluster property in each one of the two states we get

$$
\left\langle P^{2}\right\rangle=\frac{1}{2}\left\langle P^{2}\right\rangle_{\alpha}+\frac{1}{2}\left\langle P^{2}\right\rangle_{\beta}=z_{\alpha}^{2}
$$

but since $\left\langle P^{2}\right\rangle=0, z_{\alpha}=0$.

In conclusion we have shown rigorously, assuming hermiticity of $i \bar{\psi} \gamma_{5} \psi$ and using standard properties of Ginsparg-Wilson fermions, that parity is not spontaneously broken in the one-flavour model, at least for the standard order parameters.

\section{The $N_{F}$ flavours case}

We study now the case of $N_{F}$ flavours, in general with different, non-zero masses. Most of the results from the previous section apply here as well with small modifications. The fermionic action is

$$
\sum_{\alpha=1}^{N_{F}} \bar{\psi}_{\alpha}\left(\Delta+m_{\alpha}\right) \psi_{\alpha}
$$

The complete spectrum of the Dirac operator consists of $N_{F}$ copies of the single flavour spectrum, each of them calculated with the mass of the corresponding flavour.

Let us consider the usual pseudoscalar order parameter for a single flavour $\beta$, $i \bar{\psi}_{\beta} \gamma_{5} \psi_{\beta}$. The corresponding generating function $P(q)$ can be computed easily:

$$
P(q)=\left\langle\frac{\operatorname{det}\left(\Delta+m_{\beta}+\frac{q}{V} \gamma_{5}\right)}{\operatorname{det}\left(\Delta+m_{\beta}\right)}\right\rangle_{N_{F}}
$$

The average is taken over the effective gauge theory with $N_{F}$ flavours, but only the $\beta$ flavour appears within the average 1 . The calculation is identical to the one for the single flavour case and gives

$$
P(q)=\left\langle\prod_{j}\left(1+\frac{1}{\mu_{j}^{\beta}} \frac{q}{V}\right)\right\rangle
$$

The superindex on the eigenvalues indicate flavour, that is, $\mu^{\beta}$ belongs to the spectrum of $\gamma_{5}\left(\Delta+m_{\beta}\right)$. Equation (27) is essentially the same as the one for one flavour, and the moments of $P(q)$ still are, up to constants, the same as the moments of the density of topological charge $Q / V$. The only difference is that now the average is taken in the theory with $N_{F}$ flavours. This doesn't change any of the conclusions: all odd moments vanish by symmetry, the second

\footnotetext{
${ }^{1}$ Our fermionic determinant (and hence, its eigenvalues) will always refer to a single flavour.
} 
moment vanish in the thermodynamic limit because of the hermiticity of $i \bar{\psi} \gamma_{5} \psi$, the density of topological charge goes to a delta centered on the origin, and therefore all the higher moments vanish as well (when $V \rightarrow \infty$ ). This is valid for any flavour separately, and so will be valid as well for any linear combination $\sum_{\alpha} A_{\alpha} i \bar{\psi}_{\alpha} \gamma_{5} \psi_{\alpha}$. The extension to the unsubstracted order parameter is trivial.

Let us consider now the degenerate case, all flavours with equal nonzero masses. The action now enjoys flavour symmetry. As stated in the introduction of this paper, the Vafa-Witten theorem [2] for vector-like symmetries does not apply to $\mathrm{G}-\mathrm{W}$ fermions because even if the integration measure is positive definite, the Dirac operator does not anticommute with $\gamma_{5}$. However we can study this symmetry with the p.d.f. method as we did for parity.

Consider first the case of two degenerate flavours and the standard order parameters $\bar{\psi} \tau_{3} \psi$ and $i \bar{\psi} \gamma_{5} \tau_{3} \psi$. Proceeding as before we find for the first order parameter:

$$
P(q)=\left\langle\frac{\operatorname{det}\left(\Delta+m+i \frac{q}{V}\right) \operatorname{det}\left(\Delta+m-i \frac{q}{V}\right)}{\operatorname{det}(\Delta+m)^{2}}\right\rangle_{N_{F}}=\left\langle\prod_{j}\left(1+\frac{q^{2}}{\lambda_{j}^{2} V^{2}}\right)\right\rangle_{N_{F}}
$$

where $\lambda_{j}$ are the eigenvalues of $\Delta+m$. By the same argument we have use repeatedly before, $P(q) \rightarrow 1$ in the thermodynamic limit 2 .

Similarly for $i \bar{\psi} \gamma_{5} \tau_{3} \psi$ we obtain

$$
P(q)=\left\langle\frac{\operatorname{det}\left(H+\frac{q}{V}\right) \operatorname{det}\left(H-\frac{q}{V}\right)}{\operatorname{det}\left(H^{2}\right)}\right\rangle_{N_{F}}=\left\langle\prod_{j}\left(1-\frac{q^{2}}{\mu_{j}^{2} V^{2}}\right)\right\rangle_{N_{F}}
$$

The same argument applies, and we have also that $P(q) \rightarrow 1$ in the thermodynamic limit.

The calculations can be repeated easily for the unsubstracted operators, and the result is the same. We can then conclude that here is no Aoki phase for two flavours of Ginsparg-Wilson fermions with non-zero mass. This conclusion should not be surprising at all, for the spectrum of the Ginsparg-Wilson operator with a mass term is depleted of small eigenvalues $\left(\approx \frac{1}{V}\right)$.

The extension of this proof to $N_{F}$ flavours is trivial, because it is easy to see that the preceding results apply to any pair of flavours, and therefore general flavour symmetry is realized.

\section{The chiral limit}

The results of the previous sections can not be extended in a straightforward way to QCD in the chiral limit. We lose the non-trivial lower bound on the spectrum of $\Delta+m, 0<m<|\lambda|$, and the Dirac operator has exact zero modes corresponding to gauge fields with non-trivial topology.

As we can not get a definite conclusion on the realization of parity in the chiral limit from first principles, we will follow in this case the standard wisdom. Let us consider QCD with one massless flavour. In this limit the action of the model has a new symmetry, the chiral U(1) symmetry, which is anomalous because the integration measure is not invariant under chiral U(1) global

\footnotetext{
${ }^{2}$ This conclusion rests only on the bound on the eigenvalues, and not on any other specific property of the Dirac operator.
} 
transformations. The Jacobian associated to this change of variables introduce an extra term to the pure gauge action proportional to the topological charge of the gauge configuration, the $\theta$-vacuum term, which allows one to understand the absence of a Goldstone boson in the model, but this fact generates the well known strong CP problem. All these features of QCD in the continuum formulation are well reproduced in lattice QCD with Ginsparg-Wilson fermions, as discussed at the beginning of this paper.

Let's consider first the unsubstracted scalar order parameter, $\bar{\psi}\left(1-\frac{a D}{2}\right) \psi$. The corresponding generating function in the chiral limit and for a generic value of $\theta$ can be written as

$$
P(q)=\frac{\int[d A] e^{-S_{Y M}} e^{-i \theta Q} \operatorname{det}\left(D+i \frac{q}{V}\left(1-\frac{a D}{2}\right)\right)}{Z}
$$

with $Z=\int[d A] e^{-S_{Y M}} e^{-i \theta Q} \operatorname{det}(D)$ and $Q=n^{-}-n^{+}$. The contribution to the determinant in the numerator of (30) coming from pairs of complex eigenvalues of $D$ is easily computed and gives a factor

$$
f_{0}(q)=\prod_{j}\left(\left|\lambda_{j}\right|^{2}-\frac{q^{2}}{V^{2}}\left(1-\frac{a^{2}\left|\lambda_{j}\right|^{2}}{4}\right)\right)
$$

where the product is taken over all different pairs of complex eigenvalues. Each chiral mode corresponding to an eigenvalue $\frac{2}{a}$ contributes a factor of $\frac{2}{a}$ to the determinant, and each chiral mode corresponding to a zero eigenvalue contributes a factor of $i \frac{q}{V}$. The normalization factor $Z$ is of course calculated from the same expressions by setting $q=0$. Therefore we can write $P(q)$ as

$$
P(q)=Z^{-1} \int[d A] e^{-S_{Y M}} e^{-i \theta Q}\left(\frac{2}{a}\right)^{n^{\prime+}+n^{\prime-}}\left(\frac{i q}{V}\right)^{n^{+}+n^{-}} f_{0}(q)
$$

The computation for the pseudoscalar order parameter $i \bar{\psi} \gamma_{5}\left(1-\frac{a D}{2}\right) \psi$ is similar and the final result is

$$
P(q)=Z^{-1} \int[d A] e^{-S_{Y M}} e^{-i \theta Q}\left(\frac{2}{a}\right)^{n^{\prime+}+n^{\prime-}}(-1)^{n^{+}}\left(\frac{q}{V}\right)^{n^{+}+n^{-}} f_{0}(q)
$$

With the definition

$$
f_{S}(q)=\left(\frac{2}{a}\right)^{n^{\prime+}+n^{\prime-}}\left(\frac{i q}{V}\right)^{n^{+}+n^{-}} f_{0}(q)
$$

and denoting by $P_{S}(q)$ and $P_{P}(q)$ the generating functions for the scalar and pseudoscalar respectively, we can rewrite the above results in the following way

$$
\begin{aligned}
& P_{S}(q)=Z_{S}^{-1} \int[d A] e^{-S_{Y M}} e^{-i \theta Q} f_{S}(q) \\
& P_{P}(q)=Z_{P}^{-1} \int[d A] e^{-S_{Y M}} e^{-i \theta Q} f_{S}(q)(-i)^{Q}
\end{aligned}
$$

From these expressions we see that only the $Q= \pm 1$ sectors contribute to the chiral condensate $3\langle S\rangle$, and that the dependence with the parameter $\theta$ is

\footnotetext{
${ }^{3}$ In fact only a subset of the $Q= \pm 1$ configurations give a non-vanishing contribution, those with only one zero mode, that is, that verify $n^{-}+n^{+}=1$ as well as $n^{-}-n^{+}= \pm 1$. Similar considerations apply to the other cases discussed in the text.
} 
$\langle S\rangle \propto \cos (\theta)$. For the second moment $\left\langle S^{2}\right\rangle$, the only non-vanishing contributions come from the sectors with $Q=0$ and $Q= \pm 2$, and we can write

$$
\left\langle S^{2}\right\rangle=A_{0}+A_{2}
$$

where $A_{0}$ is the contribution coming from the $Q=0$ sector and $A_{2}$ the contribution coming from the $Q= \pm 2$ sector. Looking at the expression for the pseudoscalar condensate, all the odd moments vanish because of parity symmetry. For the second moment we find that

$$
\left\langle P^{2}\right\rangle=A_{0}-A_{2}
$$

Looking at moments of higher order we would find an infinite set of relations.

Since due to the chiral anomaly, strictly speaking, we do not have a new symmetry in the chiral limit of one flavour QCD, the standard wisdom is that the vacuum expectation value of the chiral order parameter will not vanish as $m \rightarrow 0$. Moreover, the absence of massless particles in the one flavour model suggests that the perturbation series in powers of $m$ does not give rise to infrared divergences [27, the free energy density is an ordinary Taylor series in $m$ [26, 27; and in what concerns the chiral condensate, the chiral and thermodynamical limits commute.

On the other hand, the free energy density of the model at $m \neq 0$ and $\theta=0$ can be computed in the thermodynamical limit from the topologically trivial sector $Q=0$ [26, 27. But since chiral symmetry in the $Q=0$ sector is not anomalous, it should be spontaneously broken in the topologically trivial sector if the chiral condensate takes a non vanishing value when approaching the chiral limit. In such a case, the value of the chiral condensate in the full theory and in the chiral limit will be related to the spectral density at the origin of eigenvalues of the Dirac operator of the topologically trivial sector by the well known Banks and Casher formula 28

$$
\langle S\rangle=-\pi \rho(0)=\Sigma_{0} .
$$

This equation provide us with a non trivial relation between the value of the scalar condensate in the chiral limit in the full theory, which gets all its contribution from the $Q=+1$ sectors, and the spectral density of eigenvalues at the origin of the Dirac operator in the topologically trivial sector $Q=0$.

The scalar condensate is invariant under parity, and therefore in the full theory in the chiral limit, irrespective of the realization of parity in the vacuum we expect its probability distribution function to be a delta function $\delta\left(c-\Sigma_{0}\right)$. Therefore, we expect for the moments

$$
\left\langle S^{n}\right\rangle=\Sigma_{0}^{n}
$$

For the second moment, this tells us that

$$
A_{0}+A_{2}=\Sigma_{0}^{2}
$$

But as previously stated, the standard wisdom tell us that the topologically trivial sector breaks spontaneously the chiral $U(1)$ symmetry, and since $A$ is exactly the second moment of $P_{\mathcal{S}}(c)$ computed in the $Q=0$ sector, we should have

$$
A_{0}=\frac{1}{2} \Sigma_{0}^{2}
$$


and therefore also

$$
A_{2}=\frac{1}{2} \Sigma_{0}^{2}
$$

But this implies that the second moment of the pseudoscalar condensate vanishes,

$$
\left\langle P^{2}\right\rangle=0
$$

By a similar argument we can see that for higher (even) moments, $\left\langle S^{2 n}\right\rangle=\Sigma_{0}^{2 n}$ implies the vanishing of the corresponding pseudoscalar moment, $\left\langle P^{2 n}\right\rangle=0$

Symmetry under parity is the only obvious reason for the vanishing of the pseudoscalar moments, and therefore the previous result strongly suggests that parity is also realized in QCD with one massless flavour.

\section{Conclusion}

Although the common lore on QCD symmetries states that parity and vectorlike global symmetries remain unbroken, no sound theoretical proof of this hypothesis has ever been presented. The arguments given by Vafa and Witten against spontaneous breaking of these symmetries in [1, 2] were questioned by several groups [3, 4, 5, 6, and now there is agreement in the scientific community on the lack of a proof for parity realization in QCD. Concerning vector-like symmetries as flavour or baryon number conservation, it must be remarked that the staggered fermion discretization is the only known lattice regularization that fulfills the initial conditions of the Vafa-Witten theorem. Indeed the theorem is not applicable neither to the Ginsparg-Wilson regularization nor to Wilson's one, widely used for lattice QCD simulations. In the first case the theorem does not apply because even if the integration measure is positive definite, the Dirac operator does not anticommute with $\gamma_{5}$. In the second case neither of the two assumptions, positivity of the integration measure and anticommutation of the Dirac operator with $\gamma_{5}$ are fulfilled. Indeed, there exists for Wilson fermions a region of parameter space where parity and flavour symmetries are spontaneously broken, the well known Aoki phase.

On the other hand the p.d.f. formalism can be used to cast some light on the old aim of understanding the realization of symmetries of QCD from first principles. In fact, we have shown in this paper that some interesting conclusions appear when we apply the p.d.f. formalism to the Ginsparg-Wilson regularization. There, we see how the more standard order parameters for parity and flavour symmetries take a vanishing vacuum expectation value for a nonzero fermion mass. This is a major result that overcomes the difficulties found by [1, 2].

\section{Acknowledgments}

This work has been partially supported by an INFN-MEC collaboration, CICYT (grant FPA2009-09638) and DGIID-DGA (grant2007-E24/2). E. Follana is supported by Ministerio de Ciencia e Innovación through the Ramón y Cajal program, and A. Vaquero is supported by the Ministerio de Educación through the FPU program. 


\section{A Spectrum of D and Related Operators}

We start with the Ginsparg-Wilson relation with $R=1 / 2$,

$$
\gamma_{5} D+D \gamma_{5}=a D \gamma_{5} D
$$

We can also choose $D$ such that

$$
\gamma_{5} D \gamma_{5}=D^{\dagger}
$$

From (44) and (45) we obtain

$$
D+D^{\dagger}=a D D^{\dagger}=a D^{\dagger} D
$$

Therefore $D$ is a normal operator, and as such has a basis of orthonormal eigenvectors. Also eigenvectors corresponding to different eigenvalues are necessarily orthogonal. From (46) it is immediate to check that the operator $V=1-a D$ is unitary, $V^{\dagger} V=I$. Therefore the spectrum of $V$ lies in the unit circle with center in the origin, and the spectrum of $D$ must then lie in the shifted and rescaled circle of radius $\frac{1}{a}$ centered in the real axis at $\left(\frac{1}{a}, 0\right)$. The possible eigenvalues of $D$ are therefore of the form

$$
\lambda=\frac{1}{a}\left(1-e^{i \alpha}\right), \alpha \in \mathbb{R}
$$

We also have the identity

$$
\lambda+\lambda^{*}=a \lambda \lambda^{*}
$$

Let $\mathbf{v}$ be an eigenvector of $D$ with eigenvalue $\lambda, D \mathbf{v}=\lambda \mathbf{v}$. Taking into account (44)

$$
D \gamma_{5} \mathbf{v}=-\gamma_{5} D \mathbf{v}+a D \gamma_{5} D \mathbf{v}=-\lambda\left(\gamma_{5} \mathbf{v}+a D \gamma_{5} \mathbf{v}\right)
$$

Therefore using (47)

$$
D\left(\gamma_{5} \mathbf{v}\right)=-\frac{\lambda}{1-a \lambda}\left(\gamma_{5} \mathbf{v}\right)=\lambda^{*}\left(\gamma_{5} \mathbf{v}\right)
$$

Thus, if $\mathbf{v}$ is an eigenvector of $D$ with eigenvalue $\lambda$, then $\gamma_{5} \mathbf{v}$ is another eigenvector with eigenvalue $\lambda^{*}$, and if $\lambda$ is not real then those two eigenvectors correspond to different eigenvalues and must be orthogonal. On the other hand if we restrict to the subspace corresponding to real eigenvalues, $\lambda=0$ or $\lambda=\frac{2}{a}, \gamma_{5}$ and $D$ commute, and therefore we can find a common basis of eigenvectors; in other words, we can find an orthonormal basis for which the eigenvectors of $D$ corresponding to real eigenvalues are chiral. If we denote by $n^{+}\left(n^{-}\right)$the number of eigenvectors of positive (negative) chirality in the subspace corresponding to $\lambda=0$, and similarly $n^{\prime+}\left(n^{\prime-}\right)$ for the subspace corresponding to $\lambda=\frac{2}{a}$, then $\operatorname{Tr}\left(\gamma_{5}\right)=0$ and $Q=\frac{a}{2} \operatorname{Tr}\left(\gamma_{5} D\right)$ imply

$$
\begin{aligned}
n^{+}-n^{-} & =n^{\prime-}-n^{+} \\
Q & =n^{-}-n^{+}
\end{aligned}
$$

We denote by $V$ the size of the matrix $D$. Then the density of topological charge, defined as $\frac{Q}{V}$, is bounded in absolute value by $1,\left|\frac{Q}{V}\right| \leq 1$. 
The operator in the fermion action is

$$
\Delta+m=\left(1-\frac{a m}{2}\right) D+m
$$

Its spectrum is trivially related to the spectrum of $D$; if $\lambda$ are as before the eigenvalues of $D$, then the eigenvalues of (53) are $\left(1-\frac{a m}{2}\right) \lambda+m$. They still lie in a circle with the center in the real axis, and the possible real eigenvalues are now $m$ and $\frac{2}{a}$. We will always require that $0<m<\frac{2}{a}$, then the operator (53) preserves the position of the higher real eigenvalue [26].

We will also need the spectrum of $H=\gamma_{5}(\Delta+m)$. It is easy to see that $H$ is an hermitian operator, $H^{\dagger}=H$, and therefore has real spectrum $\mu_{j}$. We can calculate this spectrum by noting that the matrix $\gamma_{5}(\Delta+m)$ is block diagonal in the basis of eigenvectors of $D$. If we denote by $\mathbf{v}_{\lambda}$ such and eigenvector with non-real eigenvalue $\lambda$, we have

$$
\begin{aligned}
H \mathbf{v}_{\lambda} & =\gamma_{5}\left(1-\frac{a m}{2}\right) \lambda \mathbf{v}_{\lambda}+m \mathbf{v}_{\lambda^{*}}=\left(m+\lambda\left(1-\frac{a m}{2}\right)\right) \mathbf{v}_{\lambda^{*}} \\
H \mathbf{v}_{\lambda^{*}} & =\gamma_{5}\left(1-\frac{a m}{2}\right) \lambda^{*} \mathbf{v}_{\lambda^{*}}+m \mathbf{v}_{\lambda}=\left(m+\lambda^{*}\left(1-\frac{a m}{2}\right)\right) \mathbf{v}_{\lambda}
\end{aligned}
$$

We therefore have a $2 \times 2$ block

$$
\left(\begin{array}{cc}
0 & m+\lambda\left(1-\frac{a m}{2}\right) \\
m+\lambda^{*}\left(1-\frac{a m}{2}\right) & 0
\end{array}\right)
$$

The diagonalization of this block yields a pair of real eigenvalues $\pm \mu$ with

$$
\mu^{2}=m^{2}+\lambda \lambda^{*}\left(1-\frac{a m}{2}\right)^{2}+m\left(\lambda+\lambda^{*}\right)\left(1-\frac{a m}{2}\right)
$$

For $\lambda$ real, let $\mathbf{v}_{\lambda}$ be an eigenvector of $D$ of chirality $\chi$, that is, $\gamma_{5} \mathbf{v}_{\lambda}=\chi \mathbf{v}_{\lambda}$. Then

$$
H \mathbf{v}_{\lambda}=\gamma_{5}\left(1-\frac{a m}{2}\right) \lambda \mathbf{v}_{\lambda}+m \chi \mathbf{v}_{\lambda}=\left(m+\lambda\left(1-\frac{a m}{2}\right)\right) \chi \mathbf{v}_{\lambda}
$$

That is,

$$
\mu=\left(m+\lambda\left(1-\frac{a m}{2}\right)\right) \chi
$$

More explicitly, we have $\mu=m$ and $\mu=-m$ with degeneracy $n^{+}$and $n^{-}$ respectively, and similarly $\mu=\frac{a}{2}, \mu=-\frac{a}{2}$ with degeneracy $n^{\prime+}, n^{\prime-}$.

Note that $\operatorname{det}\left(\gamma_{5}(\Delta+m)=\operatorname{det}(\Delta+m)\right.$, and therefore

$$
\prod_{j} \lambda_{j}=\prod_{j} \mu_{j}
$$

From the above calculation we also obtain immediately a bound for $\mu$ at finite mass (this was remarked in [26]):

$$
\mu^{2} \geq m^{2}
$$

Let's consider now the operator

$$
\Delta+m+\frac{q}{V} \gamma_{5}\left(1-\frac{a D}{2}\right)
$$


We are interested in its determinant. Proceeding as before, we see that it is also block-diagonal in the basis of eigenvectors of $D$, and the contribution to the determinant coming from the block corresponding to a complex pair $\mathbf{v}_{\lambda}, \mathbf{v}_{\bar{\lambda}}$ is given by

$$
\begin{array}{cc}
\operatorname{det}\left(\begin{array}{cc}
\left(1-\frac{a m}{2}\right) \lambda+m & \frac{q}{V}\left(1-\frac{a \lambda}{2}\right) \\
\frac{q}{V}\left(1-\frac{a \lambda^{*}}{2}\right) & \left(1-\frac{a m}{2}\right) \lambda^{*}+m
\end{array}\right)= \\
m^{2}+\left[1-\left(\frac{a m}{2}\right)^{2}\right] \lambda \lambda^{*}-\frac{q^{2}}{V^{2}}\left(1-\frac{a^{2} \lambda \lambda^{*}}{4}\right)
\end{array}
$$

where we have used identity (48). We also have the bound

$$
\left|\frac{1-\frac{a^{2} \lambda \lambda^{*}}{4}}{m^{2}+\left(1-\frac{(a m)^{2}}{4}\right) \lambda \lambda^{*}}\right| \leq \frac{1}{m^{2}}
$$

A chiral mode $\lambda$ with chirality $\chi$ gives a contribution

$$
\left(1-\frac{a m}{2}\right) \lambda+m+\frac{q}{V}\left(1-\frac{a \lambda}{2}\right) \chi
$$

For $\lambda=\frac{2}{a}$ the contribution is just $\frac{2}{a}$, whereas for a zero mode $\lambda=0$ with chirality $\chi$, the contribution is $m+\chi \frac{q}{V}$.

\section{References}

[1] C. Vafa and E. Witten, Phys. Rev. Lett. 53 (1984) 535.

[2] C. Vafa and E. Witten, Nucl. Phys. B 234 (1984) 173.

[3] V. Azcoiti and A. Galante, Phys. Rev. Lett. 83 (1999) 1518 arXiv:hep-th/9901068.

[4] X. Ji, Phys. Lett. B 554 (2003) 33 arXiv:hep-ph/0108162.

[5] P. R. Cromptom, Phys. Rev. D $\quad \mathbf{7 2} \quad(2005) \quad 076003$ arXiv:hep-lat/0506028.

[6] V. Azcoiti, G. di Carlo and A. Vaquero, JHEP 04 (2008) 035 arXiv:0804.1338.

[7] S. Aoki, Phys. Rev. D 30 (1984) 2653.

[8] S. Aoki, Phys. Rev. Lett. 57 (1986) 3136.

[9] V. Azcoiti, G. di Carlo and A. Vaquero, Phys. Rev. D 79 (2009) 014509 arXiv:0809.2972.

[10] S. Sharpe, Phys. Rev. D 79 (2009) 054503 [arXiv:0811.0409].

[11] V. Azcoiti, V. Laliena and X.Q. Luo, Phys. Lett. B 354 (1995) 111 arXiv:hep-th/9509091. 
[12] P.H. Ginsparg and K. G. Wilson, Phys. Rev. D 25 (1982) 2649.

[13] N.B. Nielsen and M. Ninomiya, Phys. Lett. B 105 (1981) 219.

[14] N.B. Nielsen and M. Ninomiya, Nucl. Phys. B 185 (1981) 20.

[15] P. Hasenfratz, Nucl. Phys. B(Proc. Suppl.) 63 (1998) 53 arXiv:hep-lat/9709110.

[16] H. Neuberger, Phys. Lett. B 417 (1998) 141 arXiv:hep-lat/9707022.

[17] H. Neuberger, Phys. Lett. B 427 (1998) 353 arXiv:hep-lat/9801031.

[18] P. Hasenfratz, V. Laliena and F. Niedermayer, Phys. Lett. B 427 (1998) 125 arXiv:hep-lat/9801021.

[19] K. Kikukawa and A. Yamada, Phys. Lett. B 448 (1999) 265 arXiv:hep-lat/9806013.

[20] K. Fujikawa, Nucl. Phys. B 546 (1999) 480 arXiv:hep-th/9811235.

[21] H. Suzuki, Prog. Theor. Phys. 102 (1999) 141 arXiv:hep-th/9812019.

[22] D.H. Adams, Annals. Phys. 296 (2002) 131 arXiv:hep-lat/9812003.

[23] D.H. Adams, J. Math. Phys. 42 (2001) 5522 arXiv:hep-lat/0009026.

[24] S. Chandrasekharan, Phys. Rev. $\quad$ D $\quad 60$ (1999) 074503 arXiv:hep-lat/9805015.

[25] M. Lüscher, Phys. Lett. B 428 (1998) 342 arXiv:hep-lat/9802011.

[26] F. Niedermayer, Nucl. Phys. Proc. Suppl. $\mathbf{7 3}$ (1999) 105 arXiv:hep-lat/9810026.

[27] H. Leutwyler and A. Smilga, Phys. Rev. D 46 (1992) 5607.

[28] T. Banks and A. Casher, Nucl. Phys. B 169 (1980) 103. 